\title{
Cinétique démographique des populations pré-imaginales d'Aedes mariae (Diptera : Culicidae) des côtes occidentales algériennes
}

\author{
K. Hassaïne ${ }^{1}$ \\ S. Gourmala ${ }^{1}$ \\ G. Metge ${ }^{2}$
}

Mots-clés : Aedes mariae, Culicidae, Algérie, bioécologie, ponte, éclosion, stades immatures, cycle vital.

Le complexe d'espèces jumelles mariae se développe dans les creux de rocher (rock pools) contenant de l'eau de mer. L'espèce Aedes mariae Sergent \& Sergent, 1903 se rencontre sur le pourtour du bassin tyrrhénien et notamment sur la côte rocheuse de l'ouest algérien. Le suivi durant une année de la dynamique de la population a permis de démontrer que le cycle biologique est sensiblement différent de celui observé en Méditerranée septentrionale. La présence de femelles actives aux mois de janvier et février et d'un stock réduit d'œufs dans les gîtes, montrent que la population d'Ae. mariae des côtes occidentales algériennes adopte une double stratégie de développement. Les éclosions les plus importantes sont enregistrées lorsque la conductivité est comprise entre 52.000 et $75.000 \mu \mathrm{S} . \mathrm{m}^{-1}$ résultant d'un apport d'eau de mer et de la concentration en sels sous l'effet de l'évaporation. Les conductivités supérieures à $100.000 \mu \mathrm{S} . \mathrm{m}^{-1}$, entravent l'éclosion des œufs en favorisant la dessiccation biologique.

\section{Demographic kinetics of pre-imaginal populations of Aedes mariae (Diptera : Culicidae) in west Algerian coasts}

Keywords : Aedes mariae, Culicidae, Algeria, bioecology, hatching, egg laying, preimaginal instars, life cycle.

Aedes mariae is a thalassophilic complex of three sibling species whose preimaginal instars inhabit rock-pools of the Mediterranean shores. These Aedes mariae species Sergent \& Sergent, 1903 co-occur around the Tyrrhenian basin and notably on the rocky coast of Western Algeria. During one year, a survey on the dynamics of populations showed that the biological cycle is obviously different from those observed in the Northern Mediterranean sea by the presence of active females in January and February and a reduced supply of eggs in their habits ; the Aedes mariae populations of west Algerian coasts a double strategy for development. The most important hatching recorded when conductivity ranges between 52,000 and $75,000 \mu \mathrm{m}^{-1} \mathrm{resul}^{-}$ ting from the supply of sea water and a concentration of salts from evaporation. Conductivity in excess of $100,000 \mu \mathrm{m}^{-1}$, restricts the egg hatching in favour of biological desiccation.

\section{Introduction}

La présence d'Aedes mariae Sergent \& Sergent, 1903, sur les côtes algériennes a été confirmée par les premiers travaux de Seguy (1924), Senevet \& Andarelli $(1954,1963,1964)$ et Rioux $(1958)$. Cette espèce des rock-pools du littoral méditerranéen correspondait à une seule et même espèce pour ces auteurs qui écar-

1. Université Abou Bekr Belkaïd, Faculté des Sciences, Département de Biologie, B.P. 119, 13000 Tlemcen, Algérie.

2. Faculté des Sciences St Jérôme, Aix-Marseille III. Antenne Universitaire d'Arles, Laboratoire d'Écologie, Espace Van Gogh, place Félix Rey, 13000 Arles, France. taient toute possibilité d'une subdivision biogéographique.

La démarche taxonomique, en matière d'espèces jumelles, est bien établie par les travaux se rapportant au complexe detritus (Pasteur et al. 1977, Rioux et al. 1998). Les résultats d'études cytotaxonomiques et morphotaxonomiques obtenus par Bullini \& Coluzzi (1973), Coluzzi et al. (1974b), permettent d'identifier Ae. mariae comme un complexe de trois espèces jumelles réparties selon trois zones biogéographiques bien distinctes :

- une espèce tyrrhénienne : Aedes mariae Sergent \& Sergent, 1903 ;

- une espèce adriatique : Aedes zammitii Theobald, 1903 ; 
- une espéce méridionale : Aedes phoeniciae Coluzzi et Sabatini, 1968.

La forme de la Méditerranée occidentale (France, Espagne, Maroc, Algérie, Tunisie) correspond à l'aire de répartition de l'espèce tyrrhénienne Aedes mariae (Fig.1).

$\mathrm{Au}$ cours des trois dernières décennies, les études bioécologiques concernant les Culicides du Maghreb et, plus particulièrement de l'Algérie, ont été délaissées ; les seuls travaux réalisés sur ces sujets sont des études écologiques disparates, le plus souvent non publiées. La bioécologie d'Ae.mariae sur les côtes méditerranéennes occidentales a fait l'objet de peu de travaux : Messerlin (1938), Vacelet (1959), Rioux \& Nicoli (1960). Afin d'apporter de nouvelles informations sur cette espèce, nous avons abordé l'étude dynamique des populations de la localité de Honaïne (Ouest algérien). La taille réduite des gîtes et la spécificité de leur habitat présentent de grands avantages pour une telle étude.

De 1994 à 1995, nous avons réalisé :

- une analyse démographique tenant compte de la structure de la population et de sa dynamique spatiotemporelle ;

- une recherche des facteurs écologiques clés de la cinétique démographique.

\section{Matériels et méthodes}

L'étude a été réalisée dans les rock-pools du littoral de la région de Honaine $\left(35^{\circ} 11^{\prime} \mathrm{N}, 1^{\circ} 40^{\prime} \mathrm{W}\right)$ située sur les côtes occidentales algériennes (Fig.1). La diversité physiographique des platiers rocheux délimite deux stations situées à quelques centaines de mètres l'une de l'autre. La première $(\mathrm{S} 1)$ se situe entre un et deux mètres au-dessus des plus hautes eaux et présente treize gîtes de différentes dimensions (Tableau 1) ; la seconde (S2) entre trois et quatre mètres et compte sept gîtes. Vingt gîtes ont été prospectés du 11 mars au 16 octobre1995, avec une fréquence de 7 à 15 jours.

L'évaluation de l'abondance des œufs fut réalisée mensuellement à l'aide d'un filet Langeron (vide de maille de $80 \mu \mathrm{m}$ ) ; compte-tenu de la localisation des œufs dans les anfractuosités (Coluzzi et al. 1975), il fut procédé à des raclages, à l'aide d'une brosse, sur un carré de $20 \mathrm{~cm}$ de côté, puis les œufs furent récoltés dans le filet. Le nombre de prélèvements, répartis au hasard sur chaque site, représente une surface échantillonnée d'environ $10 \%$ de la surface des gîtes. Les cufs collectés furent mis dans une cuvette, triés et comptés manuellement à l'aide de pipette puis remis dans le gîte. En période estivale, les cufs pondus à la surface de l'eau furent récoltés à l'aide du filet Langeron.

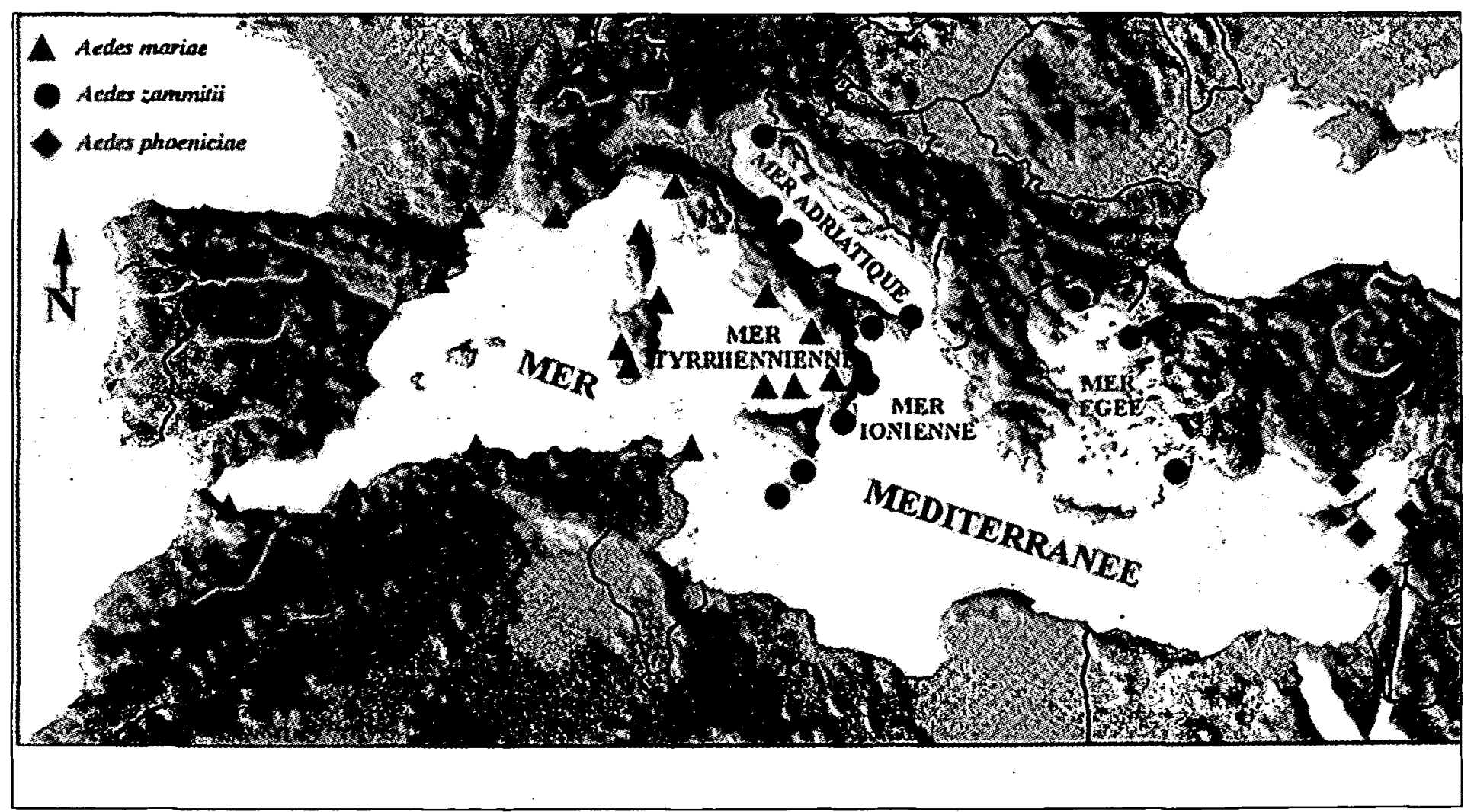

Fig. 1. Répartition du Complexe Aedes mariae dans le bassin méditerranéen.

Fig. 1. Distribution of the Aedes mariae complex in the Mediterranean basin 


\begin{tabular}{|c|c|c|}
\hline 怤 & 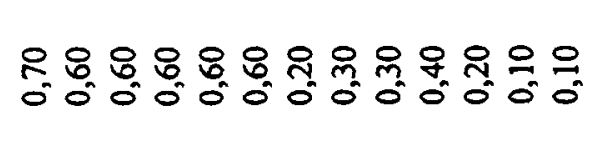 & 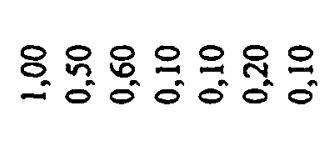 \\
\hline 吾 & 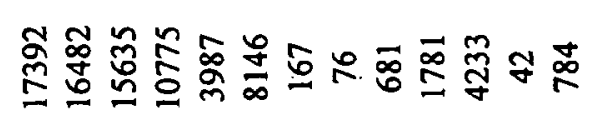 & 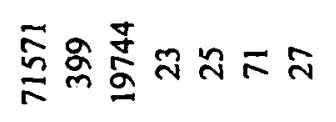 \\
\hline : & 亚令合命 & $\stackrel{\text { : }}{\mathrm{N}} 0 \stackrel{\infty}{\mathrm{n}} 0000$ \\
\hline 竞 & 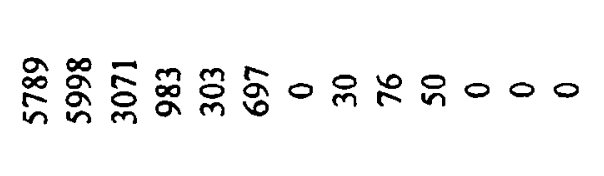 & 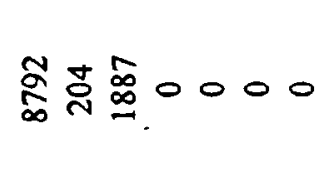 \\
\hline 言 & 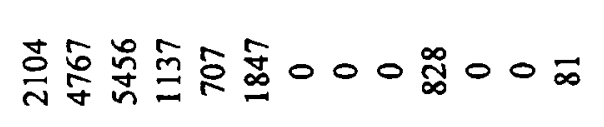 & 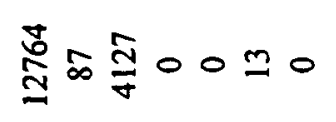 \\
\hline 产 & 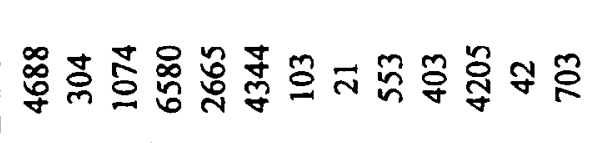 & 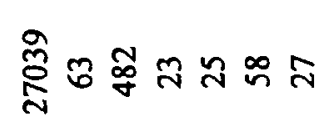 \\
\hline 至 & 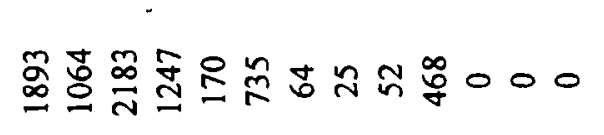 & 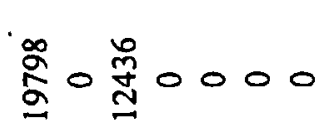 \\
\hline$\sum_{\Sigma}^{\bar{\sigma}}$ & 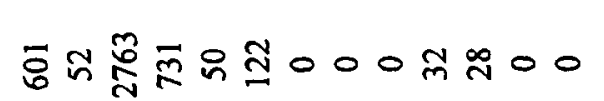 & 四 స్సి0000 \\
\hline$\frac{\bar{z}}{2}$ & 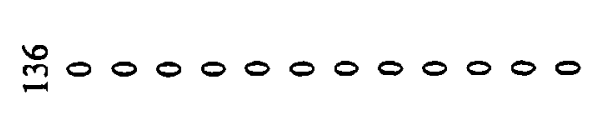 & F 000000 \\
\hline 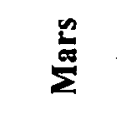 & 0000000000000 & No000000 \\
\hline 密 & 0000000000000 & N 000000 \\
\hline 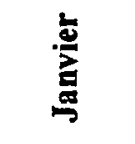 & 0000000000000 & TiNo00000 \\
\hline 绨 & 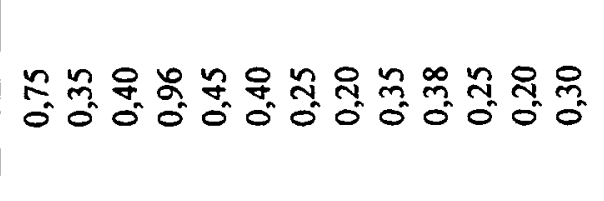 & 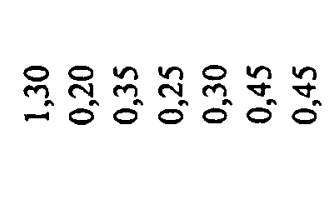 \\
\hline 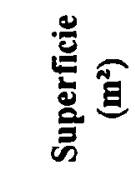 & 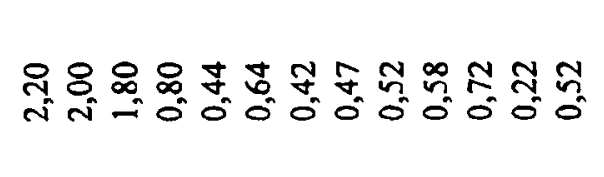 & 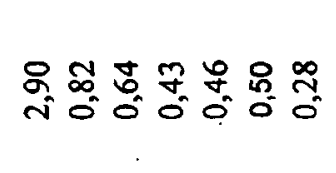 \\
\hline 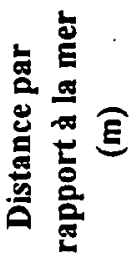 & 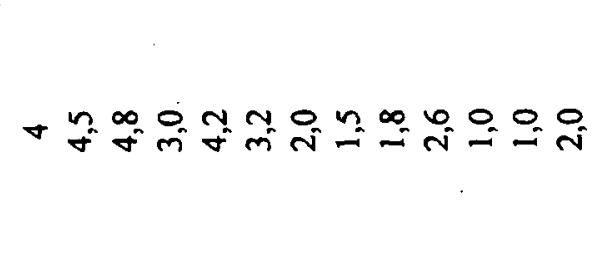 & 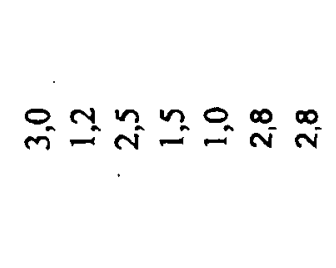 \\
\hline 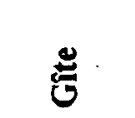 & 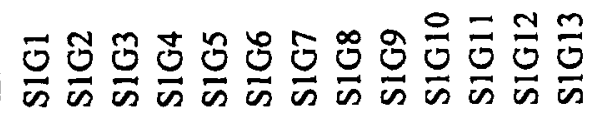 & 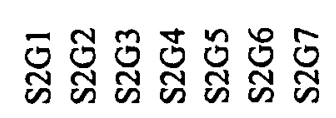 \\
\hline
\end{tabular}


Les effectifs des stades immatures - larves et nymphes - correspondent au nombre total d'individus récoltés dans chaque site à l'aide du filet Langeron. Les individus comptés in situ furent réintroduits dans le gîte.

Les paramètres physiques des creux de rocher retenus sont : la taille, la profondeur et la distance par rapport à la mer (Tableau 1).

Les paramètres physico-chimiques pris en compte sont : la conductivité, la salinité et le $\mathrm{pH}$, mesurés à l'aide d'un appareil de type SOLOMAT 2000, la température, la hauteur de l'eau. Ces mesures ont été effectuées régulièrement dans les gîtes $S 1 G 1$ et $S 2 G 1$, sites les plus représentatifs des deux stations d'étude. Ces variables écologiques sont àssociées au nombre total d'individus des stades immatures, regroupés dans un seul tableau.

Les données traitées en Analyse en Composantes Principales ACP (Thioulouse \& Chessel 1997), permettent de mettre en évidence les variables susceptibles d'influencer la dynamique des stadés immatures des populations d'Ae. mariae.

Les observations de terrain ont permis de déceler la présence de femelles en période hivernale, capturées à l'aide d'un aspirateur dans les crevasses et les petites cavités creusées dans la roche, à proximité des lieux de ponte. Quelques femelles furent capturées sur appât humain. Pour des raisons matérielles, le suivi des variations des effectifs des femelles fortement agressives, n'a pas été réalisé.

\section{Résultats}

\subsection{Effectifs des stades matures}

Nos premiers déplacements révélèrent la présence de quatre femelles actives le 14 janvier, capturées sur appât humain. Les prospections des gîtes de repos permirent la récolte de femelles dans les crevasses situées à proximité des gîtes de ponte $\mathrm{S} 1 \mathrm{G} 1, \mathrm{~S} 2 \mathrm{G} 3$ et $\mathrm{S} 1 \mathrm{G} 3$ et d'une petite grotte située à proximité du gîte $S 2 G 1$. Le nombre d'adultes capturés ne fut jamais important : un à deux individus dans quelques crevasses et deux à cinq individus dans de petites cavités rocheuses. Au total, 35 femelles ont été recensées dans ces gîtes de repos durant les mois de janvier et février. Le nombre de femelles actives augmente au cours des mois d'avril et mai où respectivement 56 et 138 individus sur appât humain furent capturés. Leur nombre s'accroît considérablement au début juin et leur évaluation précise devient alors impossible, faute de matériel adéquat.

\subsection{Effectifs d'œufs}

La plupart des gîtes sont dépourvus d'œufs durant la période défavorable allant du mois de janvier au mois d'avril, à l'exception du gîte S1G2 dans lequel 28 œufs furent récoltés dans les amas d'algues filamenteuses (Tableau 2). Dans ce même site les premières pontes eurent lieu le 17 mars . En avril, les pontes concernent aussi bien les gîtes $S 2 G 1$ que S1G1. Les autres creux de rocher sont colonisés progressivement à partir du début du mois de mai. Durant le mois de juillet, les effectifs des œufs augmentent considérablement et concernent alors l'ensemble des lieux de ponte. Elles sont importantes dans les creux situés à plus de $3 \mathrm{~m} \mathrm{du}$ front de mer pour la station 1 et $2,5 \mathrm{~m}$ pour la station 2 . Les gîtes les plus proches de la mer sont généralement très peu productifs ; l'abondance des oufs et la fréquence des pontes y sont faibles (Tableau 1).

\subsection{Variations spatio-temporelles des effectifs des larves et nymphes}

La première génération larvaire est apparue le 11 mars dans le gîte S2G1. Des éclosions de faible effectif se succèdent sur ce même site jusqu'au début de mai où se déclare la productivité de quatre autres gîtes $\mathrm{G} 1$, G3 et G6 au niveau de la station 1 ; ils sont colonisés en second (Tableau 3). Le 26 mai, douze gîtes soit plus de la moitié des gîtes, abritent des larves de différents stades. Les effectifs sont relativement faibles durant cette période printanière, et deviennent très importants en juin. Des éclosions sont enregistrées dans les creux situés à proximité de la mer principalement au cours du mois de juillet (Tableau 1). Les conditions climatiques déterminent une période d'assèchement qui se traduit par l'absence des stades larvaires et nymphaux. Sur l'ensemble des gîtes, le coup de mer du 4 août a contribué à l'apparition de nouvelles générations d'août à septembre. Le premier octobre, les éclosions nombreuses concernent 17 gîtes différents. Ensuite, les effectifs diminuent progressivement, seules douze larves de stade 4 sont dénombrées dans le gîte S2G1 le 30 octobre.

En période estivale (juillet - août), l'absence de stades larvaires et nymphaux apparût dans les gîtes les plus productifs (Fig.2). Les résultats de l'analyse en composantes principales (S1G1 et $S 2 G 1$ ), réalisée à partir de la matrice des données constituée par les six facteurs retenus et les 48 dates de prélèvements (Tableau 3), sont représentés sur la figure 3 . Pour faciliter la représentation, les plans $\mathrm{F} 1 \mathrm{xF} 2$ lignes et colonnes ont été superposés. Les axes 1 et 2 représentent des inerties respectives de $31,54 \%$ et $29,02 \%$ de la variation totale. 


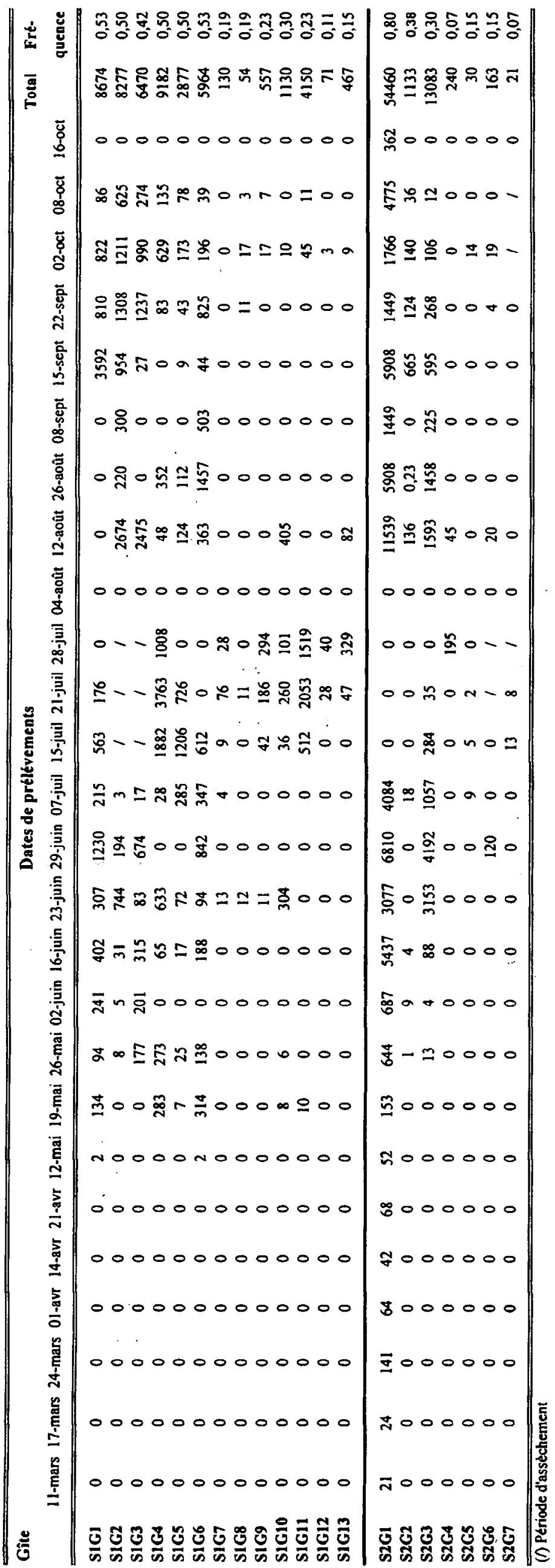




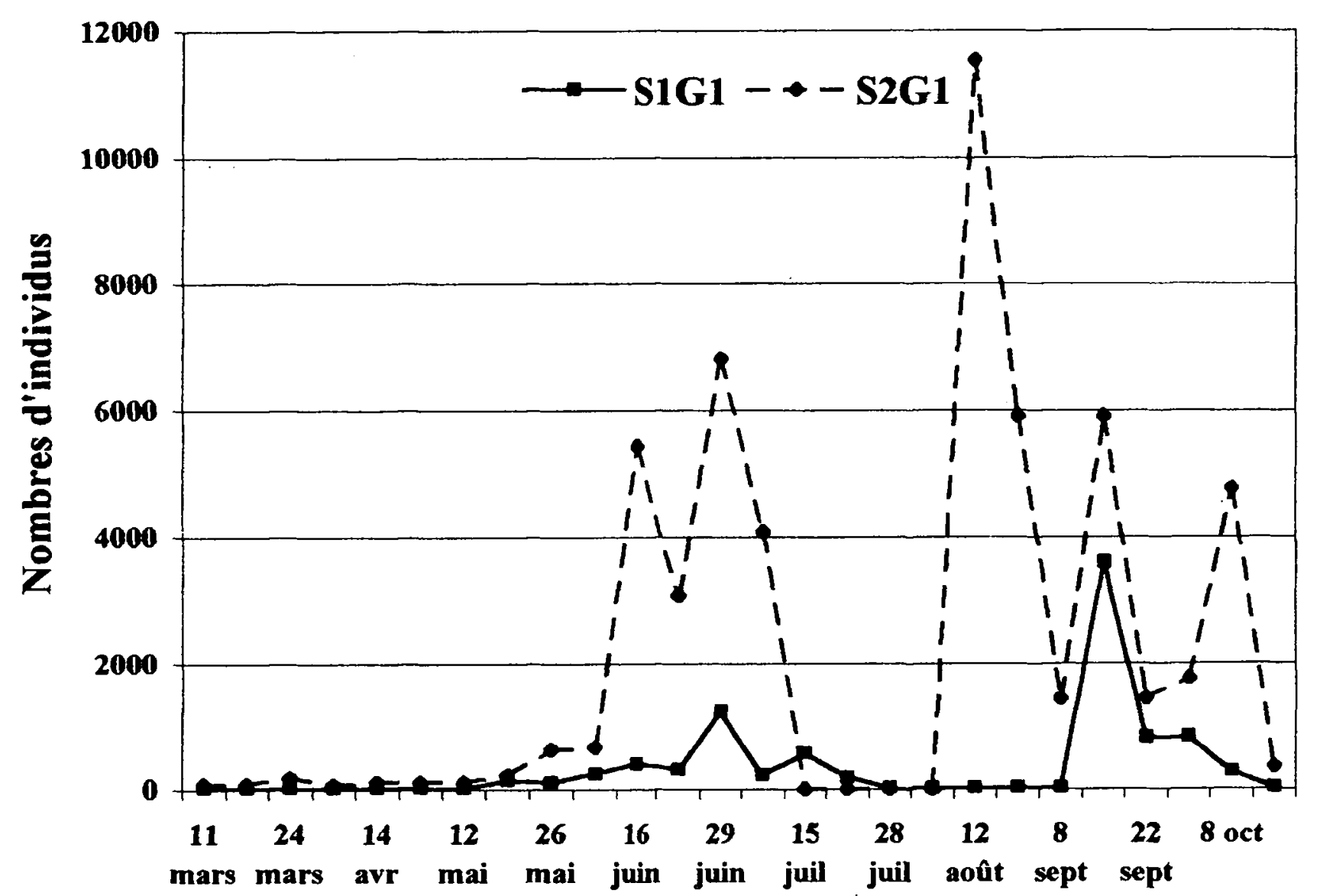

Dates de prélèvements

Fig. 2. Variation dans le temps des effectifs de larves et nymphes dans les gîtes fonctionnels S1G1 et S2G1.

Fig. 2. Temporal variations of larvae and nymphs abundance found in the functional rock-pools S1G1 and S2G1.

Sur l'axe 1, la conductivité et la salinité s'opposent à la température et la hauteur de l'eau, qui varie inversement à la conductivité et à la salinité ; ces deux facteurs s'opposent aux effectifs sur l'axe 2 . Les points "dates" (plan F1xF2) se répartissent sur l'axe 1 selon un gradient croissant d'effectifs et sur l'axe 2 selon un gradient croissant de conductivité. L'axe 1 oppose la période printanière (côté négatif) à la période estivale (côté positif). Ainsi, les points "dates" dont les effectifs sont nuls ou faibles et les valeurs de conductivité de l'ordre de $50.000 \mu \mathrm{S} . \mathrm{m}^{-1}$ s'opposent aux points représentés par les effectifs les plus élevés et les valeurs de conductivité moins importantes. Alors que ces points s'opposent, sur l'axe 2, aux points situés sur le côté positif de cet axe et traduisant des effectifs nuls et des valeurs de conductivité supérieures à $100.000 \mu \mathrm{S} . \mathrm{m}^{-1}$. Les points situés près de l'origine, se caractérisent par des valeurs d'effectifs et de conductivité moyennes.

\section{Discussion}

Les expériences réalisées en laboratoire par Coluzzi et al. (1974a, 1975) montrent que les femelles issues de larves élevées en jours longs (ce qui sur le terrain correspond aux générations estivales) pondent préférentiellement sur des surfaces d'eau libre. Les œufs ont un développement immédiat. Par contre, celles issues de larves élevées en jours courts, que l'on peut assimiler aux générations prévernales, pondent sur des surfaces humides et leurs œufs subissent une période de quiescence. Ainsi, le stock hivernal d'œufs quiescents, incrusté dans les cavités et les petites anfractuosités du littoral rocheux, bénéficie d'une protection contre les grosses vagues, en attendant des conditions favorables à l'éclosion.

Nos résultats mettent en relief trois points fondamentaux pour la période hivernale :

1) présence de femelles actives aux mois de janvier et février ;

2) absence d'œufs dans les anfractuosités : les raclages successifs à cette période n'ont pas révélé la présence d'œufs sur l'ensemble des gîtes par contre, les mêmes techniques de prélèvements ont permis de récolter des effectifs importants au printemps et en été ; 
Tableau 3. Variation temporelle des facteurs physico-chimiques et des effectifs des stades immatures.

Table 3. Temporal variations of physico-chemical parameters and number of immature instars.

\begin{tabular}{|c|c|c|c|c|c|c|c|c|c|c|c|c|c|}
\hline \multirow[t]{2}{*}{ Gîte } & \multirow{2}{*}{$\begin{array}{l}\text { Date de } \\
\text { prélévement }\end{array}$} & \multirow[t]{2}{*}{ Code } & \multirow{2}{*}{$\begin{array}{c}\text { Conductivité } \\
(\mu \mathrm{S})\end{array}$} & \multirow{2}{*}{$\begin{array}{c}\text { Salinité } \\
\text { (gfl) }\end{array}$} & \multirow[t]{2}{*}{ pH } & Temperature & \multirow{2}{*}{$\begin{array}{c}\text { Hauteur } \\
\text { (cm) }\end{array}$} & \multirow[b]{2}{*}{ Stade 1} & \multicolumn{3}{|c|}{ Nombre d'individus immatures } & \multirow[b]{2}{*}{ Nymphe } & \multirow[b]{2}{*}{ Total } \\
\hline & & & & & & $\left({ }^{\circ} \mathrm{C}\right)$ & & & Stade 2 & Stade 3 & Stade 4 & & \\
\hline \multirow[t]{24}{*}{ S2G1 } & 11-mars & $\mathrm{Tl}$ & 53470 & 37 & 7 & 17 & 55 & 10 & 11 & 0 & $\theta$ & 0 & 21 \\
\hline & 17-mars & $\mathrm{T} 2$ & 50390 & 31 & 7 & 20 & 62 & 0 & 3 & 21 & $\mathbf{0}$ & 0 & 24 \\
\hline & 24-mars & T3 & 52060 & 35 & 8,55 & 14,5 & 58 & 140 & 0 & 1 & $\mathbf{0}$ & 0 & 141 \\
\hline & 01-avi & T4 & 51330 & 34,9 & 6,8 & 14,5 & 62 & 56 & 8 & 0 & 0 & 0 & 64 \\
\hline & 14-avi & T5 & 50900 & 37,2 & 6,8 & 16 & 70 & 22 & 20 & 0 & 0 & 0 & 42 \\
\hline & 21-avr & T6 & 54540 & 38,9 & 6,8 & 16,5 & 60 & 10 & 35 & 16 & 7 & 0 & 68 \\
\hline & 12-mai & $\mathrm{T7}$ & 71110 & 45,5 & 7,5 & 20 & 55 & 0 & 14 & 7 & 30 & 1 & 52 \\
\hline & 19-mai & T8 & 67300 & 52,2 & 7,5 & 20 & 59 & 40 & 38 & 46 & 28 & 1 & 153 \\
\hline & 26-mai & T9 & 77640 & 54,2 & 8,55 & 20 & 56 & 403 & 77 & 55 & 82 & 27 & 644 \\
\hline & 02-juin & TiO & 52630 & 36,14 & 7,2 & 20 & 66,5 & 270 & 71 & 5 & 323 & 18 & 687 \\
\hline & 08-juin & $\mathrm{Tll}$ & 67350 & 48,1 & 8,5 & 24 & 43,5 & 4862 & 344 & 158 & 15 & 58 & 5437 \\
\hline & 16-juin & $\mathrm{T} 12$ & 56440 & 55,8 & 7 & 26 & 54 & 2403 & 387 & 215 & 43 & 29 & 3077 \\
\hline & 23-juin & $\mathrm{T} 13$ & 61190 & 63,1 & 7,5 & 25 & 54,5 & 5130 & 1313 & 29 & 248 & 90 & 6810 \\
\hline & 29-juin & $\mathrm{T} 14$ & 80880 & 69,3 & 7,5 & 25 & 45 & 1622 & 1227 & 336 & 630 & 269 & 4084 \\
\hline & 07-juil & T15 & 105880 & 76,58 & 7,5 & 27 & 40 & 0 & 0 & 0 & 0 & 0 & 0 \\
\hline & 15-juil & T16 & 124500 & 74,3 & 7 & 29 & 32,5 & 0 & 0 & 0 & 0 & 0 & $\mathbf{0}$ \\
\hline & 21-juil & T17 & 118000 & 71,3 & 6,2 & 26 & 28,5 & 0 & 0 & 0 & 0 & 0 & 0 \\
\hline & 28-juil & T18 & 49500 & 28,4 & 6,55 & 25 & 70 & 0 & 0 & 0 & 0 & 0 & 0 \\
\hline & 04-a00t & T19 & 60600 & 37 & 8,55 & 25 & 63,5 & 6683 & 3647 & 33 & 627 & 549 & 11539 \\
\hline & 12-aout & $T 20$ & 65150 & 59,2 & 7,5 & 27 & 53 & 3795 & 413 & 396 & 660 & 644 & 5908 \\
\hline & 19-août & T21 & 49500 & 28,4 & 7 & 23 & 71 & 531 & 0 & 9 & 135 & 774 & 1449 \\
\hline & 26-aout & $T 22$ & 53200 & 35,2 & 7,5 & 22 & 66,5 . & 831 & 580 & 251 & 52 & 52 & 1766 \\
\hline & 08-sept & $T 23$ & 62500 & 40,5 & 8,2 & 23,5 & 58 & 830 & 680 & 768 & 2205 & 292 & 4775 \\
\hline & $02-\infty c t$ & T24 & 64800 & 43,5 & 7,2 & 23 & 56 & 1412 & 406 & 247 & 318 & 142 & 2525 \\
\hline \multirow[t]{24}{*}{ S1G1 } & 11-mars & T25 & 50990 & 30 & 7 & 18 & 100 & 0 & 0 & 0 & 0 & 0 & 0 \\
\hline & 17-mars & $T 26$ & 51070 & 30,34 & 6,5 & 18,5 & 110,5 & 0 & 0 & 0 & 0 & 0 & 0 \\
\hline & 24-mars & $\mathrm{T} 27$ & 51170 & 32,24 & 7,95 & 19 & 87 & 0 & 0 & 0 & 0 & 0 & 0 \\
\hline & $01-a v r$ & $T 28$ & 50840 & 36,9 & 7,5 & 22 & 82,5 & 0 & 0 & 0 & 0 & 0 & 0 \\
\hline & 14-avr & $T 29$ & 50900 & 29,9 & 7,2 & 21 & 110 & 0 & 0 & 0 & 0 & 0 & 0 \\
\hline & $21-\mathrm{avt}$ & T30 & 55030 & 38,1 & 7 & 21,5 & 90 & 0 & 0 & 0 & 0 & 0 & 0 \\
\hline & 12-mai & $T 31$ & 60900 & 42,5 & 7,5 & 24 & 79 & 0 & 0 & 2 & 0 & 0 & 2 \\
\hline & 19-mai & T32 & 65560 & 45,3 & 7 & 25 & 74 & 28 & 8 & 13 & 18 & 67 & 134 \\
\hline & 26-mai & T33 & 73440 & 51,5 & 8,5 & 26 & 77 & 0 & 18 & 13 & 29 & 34 & 94 \\
\hline & 02-juin & T34 & 77520 & 67,3 & 8,9 & 34 & 48 & 54 & 4 & 0 & 97 & 86 & 241 \\
\hline & 08-juin & T35 & 58060 & 49,3 & 7,9 & 30 & 69 & 327 & 29 & 0 & 15 & 29 & 400 \\
\hline & 16-juin & $\mathrm{T} 36$ & 67690 & 54,03 & 7,5 & 28 & 67 & 216 & 31 & 0 & 5 & 58 & 310 \\
\hline & 23-juin & T37 & 71410 & 61,6 & 7 & 32 & 60,5 & 315 & 270 & 105 & 75 & 465 & 1230 \\
\hline & 29-juin & T38 & 76370 & 73,26 & 8,5 & 36 & 56 & 0 & 0 & 17 & 33 & 165 & 215 \\
\hline & 07-juil & T39 & 80410 & 76,81 & 7,5 & 37 & 52 & 1 & $50^{\cdots}$ & 99 & 314 & 99 & 563 \\
\hline & 15-juil & $\mathrm{T} 40$ & 108000 & 72,9 & 7 & 36 & 47,5 & 0 & 0 & 0 & 0 & 0 & 0 \\
\hline & 21-juil & T41 & 120030 & 79,7 & 7 & 24 & 43 & 0 & 0 & 0 & 0 & 0 & 0 \\
\hline & 28-juil & $\mathrm{T} 42$ & 132560 & 86,5 & 7 & 27 & 39,5 & 0 & 0 & 0 & 0 & 0 & 0 \\
\hline & 04-aout & $\mathrm{T} 43$ & 119000 & 85,4 & 7 & 26 & 34,5 & 0 & 0 & 0 & 0 & 0 & 0 \\
\hline & 12-août & $\mathrm{T} 44$ & 161000 & 88 & 6,5 & 27 & 27 & 0 & 0 & 0 & 0 & 0 & 0 \\
\hline & 19-août & $\mathrm{T} 45$ & 51000 & 32,3 & 6,8 & 29 & 76 & 0 & 0 & 0 & 0 & 0 & 0 \\
\hline & 26-200t & $\mathrm{T} 46$ & 47800 & 41,1 & 7,3 & 29 & 71 & 1534 & 866 & 0 . & 617 & 0 & 3017 \\
\hline & 08-sept & $\mathrm{T} 47$ & 74330 & 61,35 & 8,9 & 26,8 & 65,5 & 333 & 18 & 53 & 247 & 159 & 810 \\
\hline & $02-o c t$ & T48 & 67920 & 58,26 & 7 & 26,5 & 74 & 380 & 194 & 124 & 71 & 53 & 822 \\
\hline
\end{tabular}

3) présence d'une faible quantité d'œufs (une vingtaine) piégés par les amas d'algues filamenteuses du creux S2G1.

La présence d'adultes femelles en cette période montre qu'Ae. mariae des côtes occidentales algériennes a une activité semi-continue, interrompue durant les périodes froides par un repos hivernal des imagos femelles. Ceci s'explique par les valeurs relativement élevées des moyennes des minima du mois le plus froid $(\mathrm{m})$ égales ou supérieures à $9^{\circ} \mathrm{C}$. Ces températures sont compatibles avec la vie active des imagos femelles ; la photopériode peut réduire leur activité en période défavorable et contribuer au repos hivernal qui s'effectue dans les creux de rocher sous abri, à proximité des sites de ponte.

La première génération, observée le 11 mars, résulte probablement des œufs, recensés en période hivernale dans le gîte $S 2 G 1$, qui ont été pondus sur les algues et 


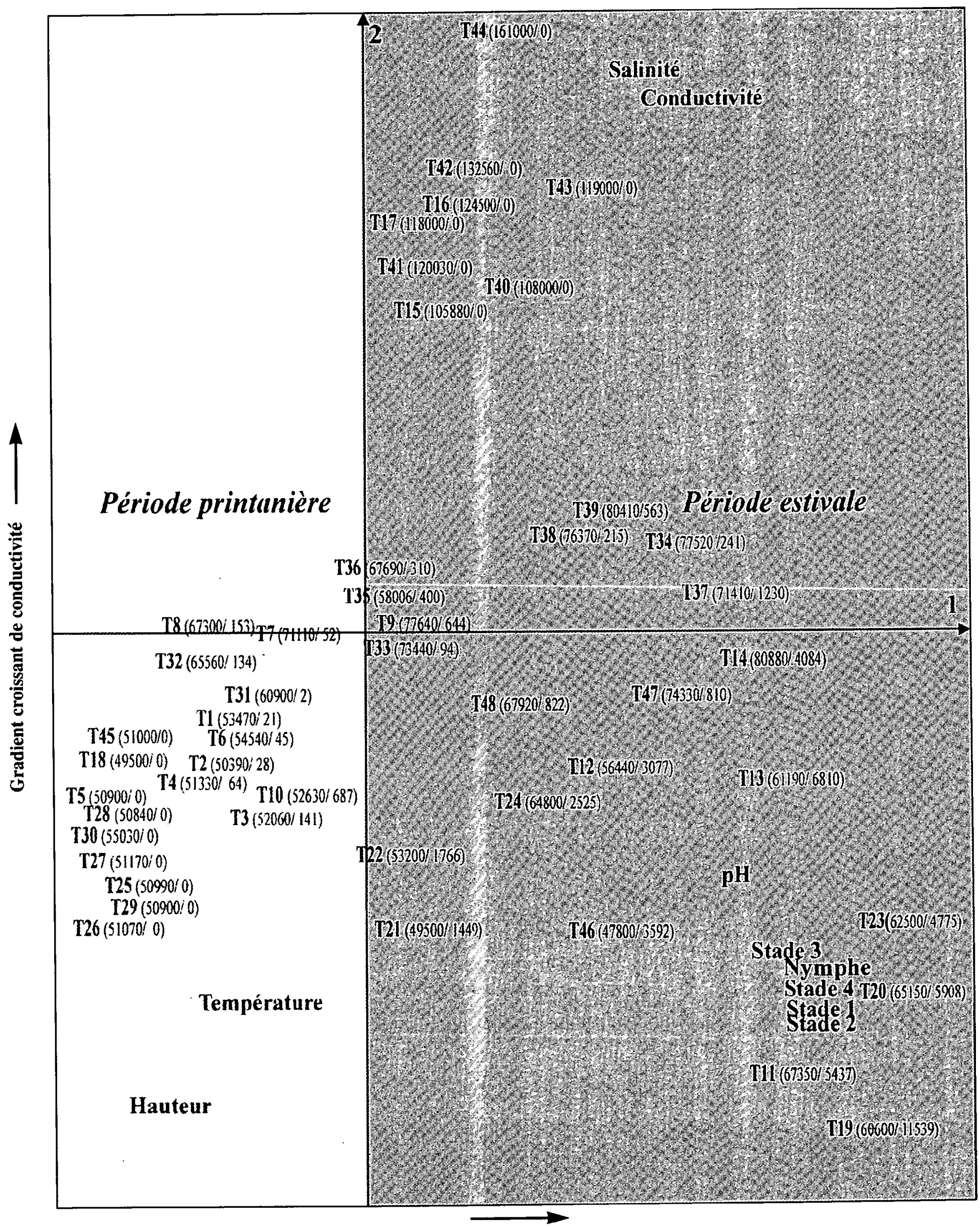

\section{Gradient croissant d'effectifs}

Fig. 3. Analyse en composantes principales mettant en relation les effectifs des stades immatures et la conductivité (plans F1 x F2 lignes et colonnes superposés).

Fig. 3. Principal Component Analysis showing the relationship between abundance of preimaginal instars and conductivity in the plane defined by the 1 st and 2 nd factors. 
sortis de la quiescence. Le stock initial d'œufs est ainsi épuisé. Les générations suivantes du 24 mars et du 21 avril proviennent d'œufs pondus par les femelles sorties de repos hivernal et que l'on a trouvées dans les lieux de repos. Ce gîte, particulier par son fonctionnement, se caractérise par une faible superficie, une profondeur relativement importante et un éloignement du front de la mer qui lui assure une protection contre les coups de mer. Ces caractéristiques en font un milieu confiné, contrairement aux autres gîtes plus ouverts.
Les autres sites deviennent fertiles au début du mois de mai grâce aux pontes récentes des femelles et non à un stock d'œufs ayant subi une diapause hivernale. À la lumière des travaux de Sinegre (1974), Guilvard \& Rioux (1986) et Metge \& Hassaïne (1998) sur Aedes caspius et Aedes detritus et de nos observations sur le terrain, il semblerait que la population d'Ae. mariae des côtes occidentales algériennes adopte une double stratégie (Fig.4) :

A En méditerranée septentrionale

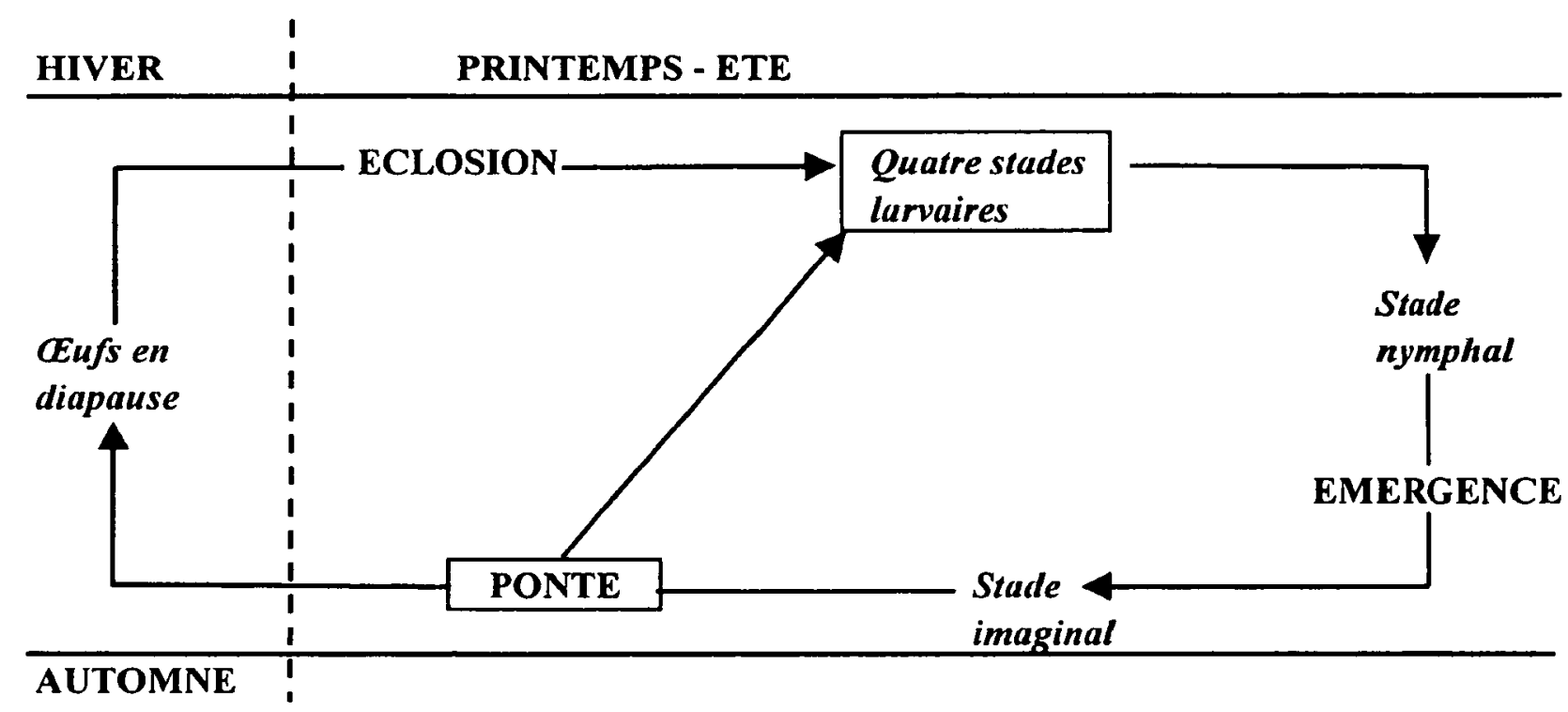

B/ Sur les côtes occidentales algériennes

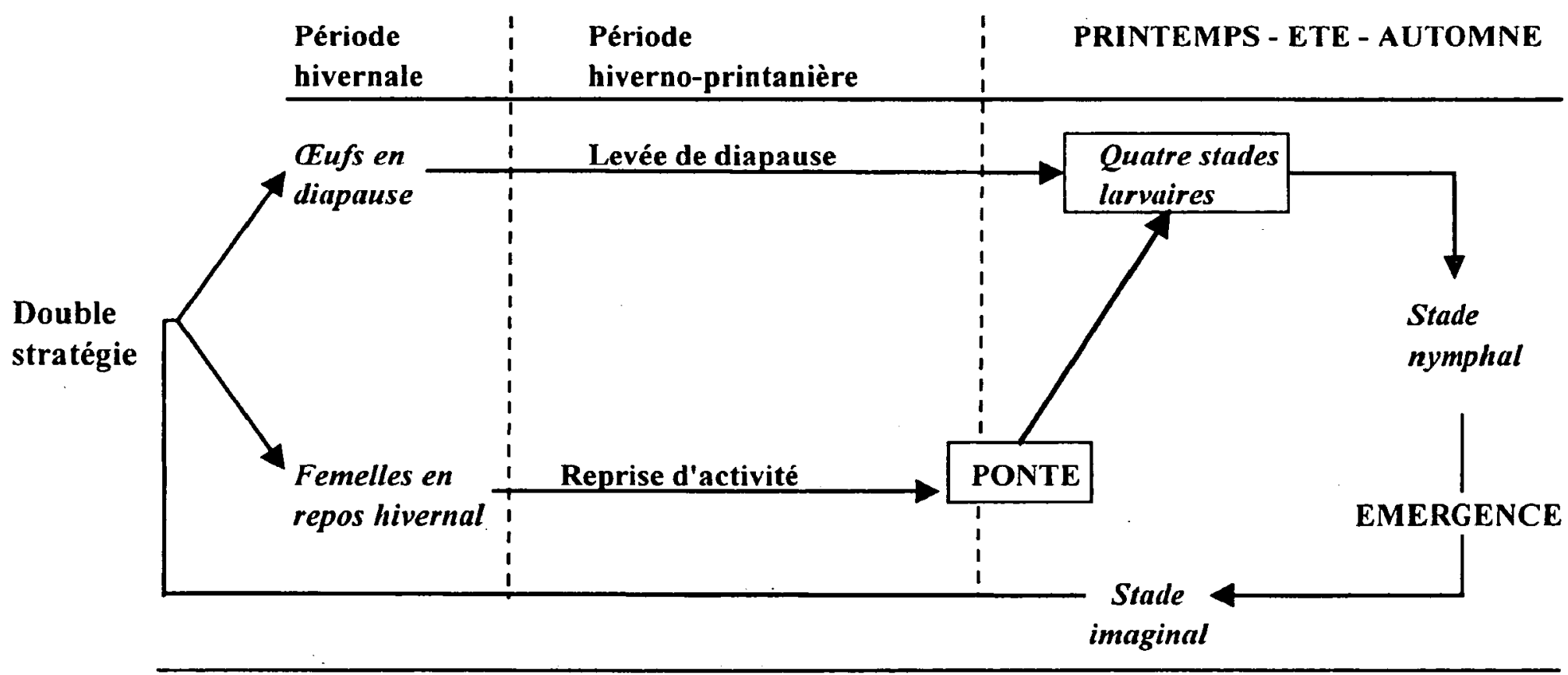

Fig. 4. Cycle de développement d'Aedes mariae.

Fig. 4. Life cycle of Aedes mariae. 
1) repos hivernal des femelles pouvant être interrompu par des conditions de photopériode et de températures favorables ;

2) nombre réduit d'œufs quiescents (Coluzzi et al. 1974a) pouvant être réactivés principalement par la longueur du jour, compte-tenu des températures moyennes minimales hivernales comprises entre $12^{\circ} \mathrm{C}$ et $16^{\circ} \mathrm{C}$.

Ce cycle biologique particulier est sensiblement différent de celui observé en Méditerranée septentrionale (Rioux 1958, Coluzzi et al. 1975) où seuls les œufs pondus en période automnale constituent le stock nécessaire à une nouvelle colonisation du milieu, lorsque les conditions deviennent favorables. Cette différence comportementale semble induite par les températures hivernales (moyennes des minima) nettement inférieures à $9^{\circ} \mathrm{C}$ sur les côtes italiennes et françaises.

En période estivale, l'absence de larve et de nymphe dans certains gîtes est liée au caractère temporaire de ces biotopes. Compte-tenu de leur situation (Tableau 1), l'apport d'eau de mer est peu fréquent, eu égard aux pertes importantes par évaporation et infiltration. Ces gîtes se caractérisent par des périodes d'assèchement de deux à quatre mois; la remise en eau provoque une réactivation des œufs et l'apparition de nouvelles générations (Tableau 2).

Dans les lieux de ponte situés à proximité de la mer, la dynamique du plan d'eau interdit la ponte et le développement larvaire durant de longues périodes, à l'exception des périodes de calme relativement courtes de 3 à 4 semaines et très vite interrompues par les coups de mer estivaux. Ces derniers provoquent des mouvements de la masse d'eau sans cesse renouvelée, donnant ainsi un caractère rhéophile à ces gîtes à submersion permanente mais fortement instables.

Dans les gîtes les plus stables et à submersion permanente, les fortes mortalités d'été des stades immatures sont consécutives à une diminution trop rapide du volume d'eau qui engendre de fortes teneurs en sels se traduisant par une conductivité élevée. Les apports en eau - pluies et / ou coups de mer - diluent les sels et favorisent d'autres éclosions ainsi que le développement des larves "rescapées". Les éclosions sont enregistrées pour des valeurs de conductivité comprises entre 50.000 et $100.000 \mu \mathrm{S} . \mathrm{m}^{-1}$. Les conductivités supérieures à $100.000 \mu S . \mathrm{m}^{-1}$ traduisent une forte teneur en sels qui provoque une importante mortalité des stades immatures et inhibent l'éclosion des œufs. Toutefois, ces teneurs n'interdisent pas les pontes sur les plans d'eau. À la remise en eau, se produisent de nombreuses éclosions après dilution des sels. Cette stase du déve- loppement des œufs est due aux fortes teneurs en sels qui bouleversent l'équilibre osmotique. En effet, la teneur en $\mathrm{NaCl}^{-}$des œufs correspond à environ $30 \mathrm{~g} / \mathrm{l}$, ce qui favorise la déperdition de l'eau contenue dans l'œuf lorsque le milieu extérieur est sursaturé. La dessiccation induite par ce processus se répercute alors sur l'éclosion des œufs. Ce phénomène similaire à la mise à sec nécessaire à l'éclosion des œufs d'Ae. caspius et $\mathrm{d}^{\prime} A e$. detritus, décrite par Sinegre (1979), contribue à leur réactivation alors synchrone, ce qui explique les explosions démographiques "enregistrées" après les dilutions.

Ainsi, en période estivale, l'élévation sensible de la teneur en sels que traduit une conductivité élevée provoque une forte mortalité des populations pré-imaginales et inhibe l'éclosion des œufs, ce qui ultérieurement contribue à la réactivation d'une manière significative et synchrone.

\section{Travaux cités}

Bullini L. \& Coluzzi L. 1973. — Electrophoretic studies ou gene-enzyme systems in mosquitoes (Diptera, Culicidae). Parasitologia, $15: 223-248$.

Coluzzi M. \& Sabatini A. 1968. - Divergenze morphologiche e barriere di sterilità nel complesso Aedes mariae (Diptera, Culicidae). Rev. Parasit., 39 (1): 49-70.

Coluzzi M. \& Bullini L. 1971. - Enzyme variants as markers in the study of precopulatory isolating mechanisms. Nature, 231 : 445-456.

Coluzzi M., Gironi M-A. \& Muir D-A. 1970. - Ulteriori esperimenti d'incrocio tra le forme del complesso mariae del genere Aedes. Parasitologia, 12 (2/3) : 119-123.

Coluzzi M., Dideco M. \& Gironi M-A., 1974a. - Embryonic diapause in Aedes mariae and changes in the oviposition behaviour induced by photoperiod. In $3^{\text {rd }}$ International Congress of Parasitology. Munich. Vol. II, Facta. Publ., : 902-903.

Coluzzi M., Sabatini A., Bullini L. \& Ramsdale C. 1974b. — Nuovi dati sulla distributione delle specie del complesso mariae del genere Aedes. Rev. Parasit., 35 (4) : 321-330.

Coluzzi M., Dideco M. \& Gironi A-M. 1975. - Influenza del fotoperiodo sulta scelta del luogo di ovideposizione in Aedes mariae (Diptera, Culicidae). Parasitologia, 17 (3) : 121-130.

Guilvard E. \& Rioux J-A. 1986. - Dynamique de l'autogénèse dans les populations naturelles d'Aedes (O.) detritus (Haliday, 1833) espèce jumelle $A$ (Diptera, Culicidae) en Camargue. Rôle prééminent de la photopériode. Ann. Parasit. Hum. Comp., 61 : 109-119.

Messerlin A. 1938. - Aedes mariae au Maroc occidental. Bull. Soc. Path. Exot., 31 (2) : 110-115.

Metge G. \& Hassaine K. 1998. - Study of environmental factors associated with oviposition by Aedes caspius and Aedes detritus along a transect in Algeria. J. AMCA, 14 (3) : 283-288.

Pasteur N., Rioux J-A, Guilvard E., Pech-Périère J. \& Verdier J-M. 1977. - Existence chez Aedes (Ochlerotatus) detritus (Haliday, 1833) (Diptera, Culicidae) de Camargue, de deux formes sympatriques et sexuellement isolées (espèces jumelles). Ann. Parasito. Hum. Comp., 52 : 325-337.

Rioux J-A. 1958. — Les Culicides du midi-méditerranéen. Encycl. Entomol. 35 P. Lechevalier, Paris : 303 p. 
Rioux J-A. \& Nicoli M. 1960. - Sur l'écologie des diptères Culicides. IV. Tolérance des larves d'Aedes mariae Sergent aux variations de concentration haline. Bull. Soc. Hist. Nat. Hérault : 21-25.

Rioux J-A., Guilvard E. \& Pasteur N. - Description d'Aedes (Ochlerotatus) coluzzi n.sp. (Diptera, Culicidae) espèce jumelle $A$ du complexe detritus. Parasitologia, $40: 353-360$.

Seguy G. 1924. - Les moustiques de l'Afrique mineure, de l'Egypte et de la Syrie. Encycl. Entomol. 24 P. Lechevalier, Paris : 257 p.

Senevet G. \& Andarelli L. 1954. - Le genre Aedes en Afrique du Nord. Arch. Inst. Pasteur, 41 : 115-141.

Senevet G. \& Andarelli L. 1963. - Les moustiques de l'Afrique du Nord et du bassin méditerranéen. III- Les Aedes. Première partie : généralités. Arch. Inst. Pasteur Algérie, 41 : 115-141.

Senevet G. \& Andarelli L. 1964. - Les moustiques de l'Afrique du Nord et du bassin méditerranéen. III- Les Aedes. Deuxième partie : description des espèces : sous genre Ochlerotatus, groupe E. Arch. Inst. Pasteur Algérie, 42 : 94-144.
Sergent E.D. \& Sergent ET. 1903. - Observations sur les moustiques des environs d'Alger. Annal. Inst. Pasteur Algérie, 17 : 60-67.

Sinegre G. 1974. - Contribution à l'étude physiologique d'Aedes (Ochlerotatus) caspius (Pallas, 1771) (Nematocera, Culicidae). Eclosion, dormance, développement, fertilité. Thèse d'Etat Univ. Languedoc : $285 \mathrm{p}$.

Sinegre G. 1979. - La chute de la teneur en ox ygène dissous à l'interface eau-sol des marais temporaires gouverne l'éclosion des œufs quiescents de moustiques. Bull. Soc. Ecophys., 4 (1) : 53-56.

Theobald F-W. 1903. - Amonograph of the Culicidae or mosquitoes. Brit. Museum. Nat. Hist., London : 252 p.

Thioulouse J. \& Chessel D. 1997. - ADE-4. A multivariate analysis and graphical display software. Statistics Comput., 7 (1) : 75-83.

Vacelat E. 1959. - Etude physico-chimique des flaques supra-littorales à salinité variable. Relations avec le peuplement. Recueil Trav. Stat. Marit. Endoume, $29: 33-38$. 